\title{
Chronic Pancreatitis in Mice by Treatment with Choline-Deficient Ethionine-Supplemented Diet
}

\author{
Satoshi IDA ${ }^{1,2)}$, Masaki OHMURAYA ${ }^{1,3)}$, Masahiko HIROTA ${ }^{4)}$, Nobuyuki OZAKI ${ }^{2)}$, \\ Sayaka HIRAMATSU ${ }^{1)}$, Hitoshi UEHARA ${ }^{1)}$, Hiroshi TAKAMORI ${ }^{2)}$, \\ Kimi ARAKI ${ }^{1)}$, Hideo BABA ${ }^{2)}$, and Ken-ichi YAMAMURA ${ }^{1)}$ \\ ${ }^{1)}$ Insitute of Resource Development and Analysis, Kumamoto University, Kumamoto 860-0811, Japan, \\ ${ }^{2)}$ Department of Gastroenterological Surgery, Kumamoto University Graduate School of Medical \\ Sciences, Kumamoto University, Kumamoto 860-8556, Japan, ${ }^{3)}$ Priority Organization for \\ Innovation and Excellence, Kumamoto University, Kumamoto 860-0811, Japan, and \\ ${ }^{4)}$ Department of Surgery, Kumamoto Regional Medical Center, Kumamoto 860-0811, Japan
}

\begin{abstract}
Although chronic pancreatitis is a risk factor for pancreatic ductal adenocarcinoma (PDA), the relationship between chronic pancreatitis and PDA remains obscure. A critical obstacle to understanding the role of chronic pancreatitis is the lack of animal models. To develop one such model, mice were fed long-term with a choline deficient ethioninesupplemented (CDE) diet. Histological evaluation revealed that chronic pancreatitis, characterized by acinar atrophy, fibrosis and well-developed tubular complexes (TCs), was observed after 24 weeks of $C D E$ diet treatment. Furthermore, expression of epidermal growth factor receptor (EGFR) and its ligands; serine protease inhibitor Kazal type 3 (Spink3) and transforming growth factor $\alpha$ (TGF $\alpha$ ) and activation of K-Ras (GTP-Ras formation), which are frequently observed in human PDA, were indeed observed in parallel with TCs formation. Neoplastic lesions were not found after 54 weeks of treatment, suggesting that a continuation of CDE diet or another insult is required for the development of PDA.
\end{abstract}

Key words: CDE diet, chronic pancreatitis, EGFR, Spink3, tubular complex

\section{Introduction}

Several recent reports have shown that chronic pancreatitis is a risk factor for pancreatic ductal adenocarcinoma (PDA) [25, 27]. This was clearly demonstrated in cases of hereditary chronic pancreatitis [26]. The incidence of pancreatic cancer in such patients increased 53 times more than that of the control [26]. However, information regarding the mechanisms behind the development of pancreatic carcinoma in the setting of chronic pancreatitis is scarce, particularly with respect to early molecular events. Concerning the molecular events in pancreatic adenocarcinoma and dysplasias, there are evidences for overexpression of epidermal growth factor receptor (EGFR) [32] and enhanced autocrine epidermal growth factor (EGF)-family signaling $[4,13,19,33,38]$. Consistent with the existence of such an autocrine loop, pancreatic adenocarcinomas overexpress EGF-family ligands, such as transforming growth factor $\alpha($ TGF $\alpha)$ and EGF $[13,19]$. EGFR and human

(Received 17 November 2009 / Accepted 3 January 2010)

Address corresponding: K. Yamamura, Division of Developmental Genetics, Institute of Molecular Embryology and Genetics, Kumamoto University, Kumamoto 860-0811, Japan 
epidermal growth factor receptor 2 (HER2) induction also occurs in low-grade pancreatic intraepithelial neoplasms (PanINs), indicating that autocrine EGF family signaling is operative in the early stages of a pancreatic neoplasm [10]. However, it is not known whether such molecular events start during the onset of pancreatic carcinoma or chronic pancreatitis.

A critical obstacle to understanding the role of chronic pancreatitis in the development of pancreatic carcinoma is the lack of appropriate animal models for chronic pancreatitis which demonstrate similar clinical courses as in human patients. Cerulein (a cholecystokinin analog) can cause supramaximal stimulation of secretion and thus cause acute pancreatitis in the mouse [21]. Repetitive acute injury to the pancreas by cerulein in the mouse can reproduce some of the morphological characteristics of human chronic panreatitis such as acinar cell atrophy and fibrosis [29]. However, analysis of carcinogenesis-related molecules has not yet been done. Several transgenic models for pancreatitis have been also described $[1,9,11,28]$. Two of them are based on genetic alterations found in human hereditary pancreatitis including the cationic trypsinogen gene and the cystic fibrosis transmembrane regulator gene $[1,11]$, but these models are limited by low penetrance ( $40 \%$ at 1 year of age) and significant latency of the phenotype. Marrache et al. [28] reported that the pancreas was atrophic and occasionally showed dilation of the pancreatic and biliary ducts secondary to proximal fibrotic stenosis in ElasshIL-1 $\beta$ (elastase promoter-driven human interleukin$1 \beta)$ transgenic mice. However, forced expression of IL- $1 \beta$ does not reflect the situation in human patients.

Chronic pancreatitis is characterized by parenchymal changes including inflammation, fibrosis and loss of exocrine and endocrine tissue. Furthermore, acinar cells are replaced by metaplastic ductal lesions, called tubular complexes (TCs), which form complex arrangements with a monolayer of flattened duct-like cells $[17,22,39]$. Clinically, pain, maldigestion and diabetes mellitus occur as the result of long-standing pancreatic injury. Choline deficient ethionine-supplemented (CDE) diet is also known to induce acute hemorrhagic pancreatitis in mice $[7,14,16,30]$. CDE diet-induced pancreatitis is considered to be an ideal model because of a similar natural history to that of the human disease as well as the histo- logical and biochemical similarities [2]. However, there are no reports on CDE diet-induced chronic pancreatitis. In the cerulein model, cerulein should be injected every hour for six hours, followed by repeated injections twice every week for 10 weeks [29]. In contrast, feeding with $\mathrm{CDE}$ diet is easy.

In this manuscript, we used long-term administration of a CDE diet to develop a model of chronic pancreatitis and examined whether increased expression of carcinogenesis-related molecules was observed during chronic pancreatitis. This CDE diet model reproduced three key responses of human chronic pancreatitis: development of acinar atrophy, fibrosis and TCs which indicated that acinar cells acquired ductal cell characteristics. We also showed increased expression of carcinogenesis-related molecules.

\section{Materials and Methods}

\section{Mice}

Female C57BL/6J mice (starting at 8 weeks old) were housed in a climate-controlled room on a 12-h light-dark cycle (CLEA Japan, Inc., Tokyo, Japan). All procedures were approved by the Animal Care and Use Committee of Kumamoto University.

\section{CDE diet-induced pancreatitis}

Acute pancreatitis was induced by a CDE diet which was purchased from MP Biomedicals (catalog number; 960214, Solon, OH, USA), including vitamin free casein (10\%), alpha soy protein (10\%), DL-ethionine $(0.5 \%)$, sucrose $(56 \%)$, lard (20\%) and mineral mix (3.5\%).

Chronic lesions were induced by repeated acute pancreatitis episodes. Mice were fasted for $24 \mathrm{~h}$ (day 0 ) and then fed a CDE diet for $72 \mathrm{~h}$ (days 1 to 4 ; necrosis phase). Then, they were fed regular laboratory chow for $72 \mathrm{~h}$ (days 4 to 7 ; regeneration phase). We defined these 7 days as one cycle and tried to induce chronic pancreatitis by repeating the cycle (Fig.1A). Mice were sacrificed on days $0,1,4,5$ and 7 and pancreatea were rapidly extracted and prepared for the following studies on weeks $0,4,12,16,24,32$, and 54 after administration of the $\mathrm{CDE}$ diet (CDE diet group, $\mathrm{n}=3$ per each period). As a control, we prepared age matched C57BL/6J mice fed regular laboratory chow (control diet group). 


\section{Histological analysis}

Pancreatic tissues were fixed by $4 \%$ paraformaldehyde for $48 \mathrm{~h}$, embedded in paraffin, sectioned, and stained with hematoxylin and eosin (HE) and azan staining. TCs were counted in $\times 100$ high-power field per cut, 5 cuts per mouse. The area of the pancreas occupied by acinar cells was measured in whole pancreas on HE slides using ImageJ software (http://rsbweb.nih.gov/ij/).

\section{Serum amylase activity}

Mouse blood samples were collected on days $0,1,4$, 5 , and 7. Serum amylase levels were measured by SRL Inc., Tokyo, Japan. Substrate for measurement of pancreatic amylase activity was 2-chloro-4-nitrophenyl-4galactopyranosylmaltoside (Gal-G2-CNP) (CicaLiquidN p-AMY, Kanto Chemical Co., Inc., Tokyo, Japan).

\section{Western blot analysis}

Pancreas samples were homogenized in lysate buffer (50 mM Tris- $\mathrm{HCl}, \mathrm{pH} 7.4,150 \mathrm{mM} \mathrm{NaCl}, 1 \%$ Nonidet $\mathrm{P}-40$, a protease inhibitor cocktail (1:100 dilution; SigmaAldrich, Tokyo, Japan) and a phosphatase inhibitor cocktail (1:100 dilution; Nacalai Tesque, Inc., Kyoto, Japan). Extracts ( $15 \mu \mathrm{g}$ of protein per lane) underwent polyacrylamide gel electrophoresis and were transferred to Immobilon polyvinylidene difluoride membranes (Millipore, Billerica, MA, USA). After $1 \mathrm{~h}$ of incubation at room temperature in blocking buffer (TBS, $5 \%$ non-fat dry milk, $0.1 \%$ Tween-20), the membranes were incubated overnight at $4{ }^{\circ} \mathrm{C}$ with primary antibodies to the antigens diluted in wash buffer (TBS, $0.1 \%$ Tween-20) containing $5 \%$ non-fat dry milk. Primary antibodies used were as follows: rabbit anti-mouse EGFR antibody (Cell Signaling Technology, Inc., Beverly, MA, USA), 1:1,000; rabbit anti-mouse phosphorylated-EGFR antibody (Cell Signaling Technology), 1:500; rabbit anti-mouse actin antibody (Sigma-Aldrich Corp., St. Louis, MO, USA), 1:1,000; rabbit anti-mouse serine protease inhibitor Kazal type 3 (Spink3) antibody [31], 1:1,000; goat anti-mouse amylase antibody (Santa Cruz Biotechnology, Inc., Santa Cruz, CA, USA), 1:2,000; rabbit anti-mouse trypsinogen antibody (Nordic Immunological Laboratories, Tilburg, Netherlands), 1:1,000; rabbit anti-mouse cyclooxygenase (Cox) 2 antibody (Cayman Chemical, Ann Arbor, MI, USA), 1:1,000; goat anti-mouse EGF antibody (R\&D systems,
Inc., Minneapolis, MN, USA), 1:1,000; and mouse antimouse TGF $\alpha$ antibody, 1:1,000 (Thermo Fisher Scientific, Fremont, CA, USA). In addition, the EZ-Detect Ras activation kit (Pierce Biotechnology, Rockford, IL, USA) was used to measure Ras activation. Membranes were then washed three times and incubated with secondary antibody [anti-rabbit immunoglobulin G antibody, 1:2,500 dilution (Amersham Biosciences Corp., Piscataway, NJ, USA) or anti-goat immunoglobulin G antibody, 1:5,000 dilution (Chemicon International, Inc., Billerica, MA, USA)] diluted in the antibody buffer for $1 \mathrm{~h}$ at room temperature, followed by three washes. Blots were developed with ECL Plus reagents (GE Healthcare, Buckinghamshire, UK). The band intensities were quantified by densitometry using ImageJ software, then normalized by the actin signals $(n=3)$.

\section{Statistical analysis}

All data are expressed as mean \pm standard error (SE). Statistical significance was determined by analysis of variance (ANOVA), followed by Dunnett's test. The differences were considered to be statistically significant at $P<0.05$.

\section{Results}

\section{Histological changes of the pancreas in the acute phase} after CDE diet

To analyze the initial reaction of the pancreas to the CDE diet, we examined histological changes during the one course of CDE diet treatment. As shown in Fig. 1B, evidence of acute pancreatitis, namely acinar cell necrosis with inflammatory infiltrates and interstitial edema, was induced after $72 \mathrm{~h}$ of CDE diet treatment. However, these pathological changes were restored to almost the normal state after the re-introduction of a normal diet (Fig. 1B). We also analyzed the serum amylase levels. The mean serum amylase level was $667 \pm 33 \mathrm{U} / 1$ on Day $0,1,067 \pm 317 \mathrm{U} / 1$ on Day 1, 2,067 $\pm 252 \mathrm{U} / 1$ on Day 4 and $683 \pm 83 \mathrm{U} / 1$ on Day 7. On day 4 , a significant elevation in the serum amylase levels was observed compared with level on Day $0(P<0.05)$ and returned to the basal level on Day 7. These results suggest that destruction and regeneration of pancreas occurred in one week. 
(A)

(B)
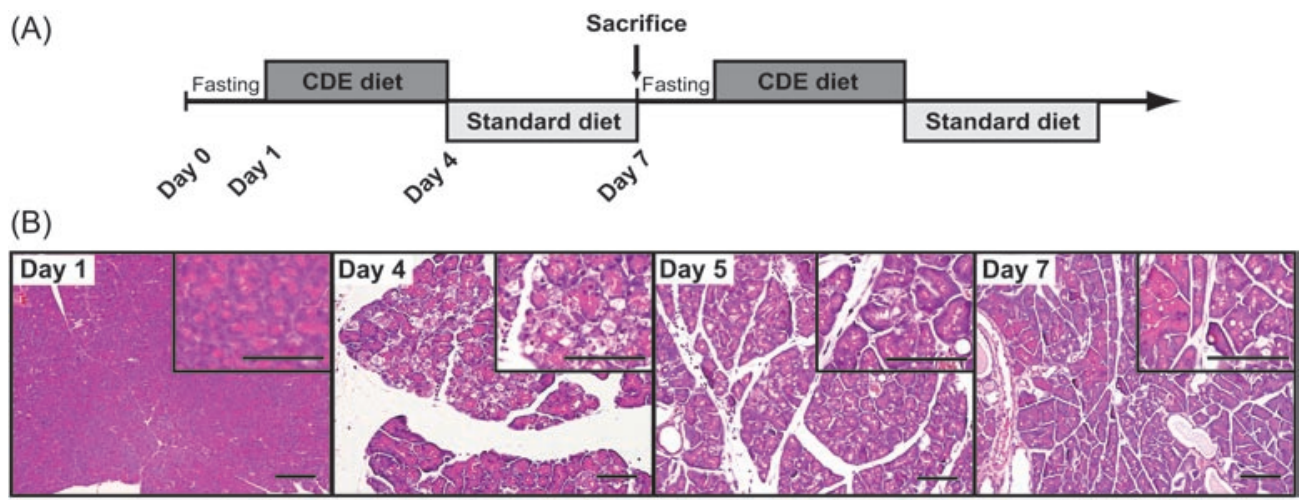

(C)

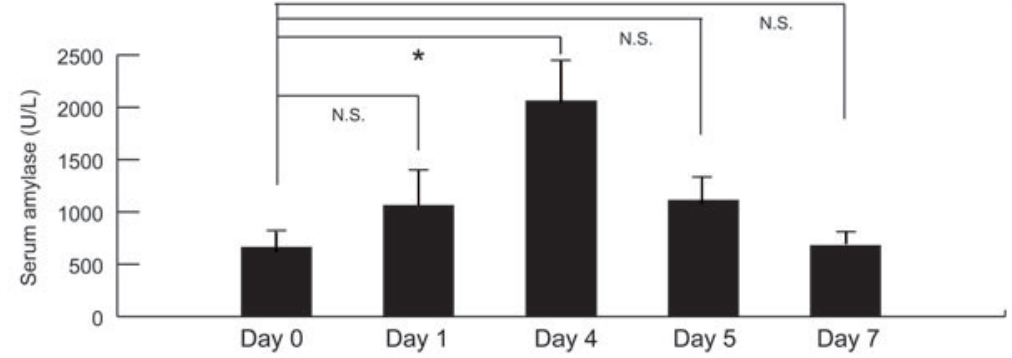

Fig. 1. Experimental protocol and histological changes of the pancreas in acute phase after one course of $\mathrm{CDE}$ diet treatment. A. Protocol for CDE diet. Mice were fasted for $24 \mathrm{~h}$ (Day 0) and then fed with a CDE diet for $72 \mathrm{~h}$ (Days 1 to 4; necrosis phase). Then, they were fed with regular laboratory chow for $72 \mathrm{~h}$ (Days 4 to 7; regeneration phase). B. Histological changes on Days 1, 4, 5, and 7. Scale bars: $100 \mu \mathrm{m}$. C. Serum amylase levels on Days 1, 4, 5, and $7(\mathrm{n}=3)$. Data represent mean \pm SE. ${ }^{*} P<0.05$.

Histological changes of the pancreas in the chronic phase after long-term CDE diet

We carefully analyzed the formation of TCs as well as acinar cell atrophy and fibrosis. Four (Fig. 2B) and twelve weeks (not shown) after starting the CDE diet, the histology was almost normal. After 16 weeks (Fig. 2C), TCs were formed in some areas. After 24 weeks, formation of TCs were remarkable (Fig. 2D and 2E). At this stage, chronic pancreatitis-like features characterized by well-developed TCs, acinar atrophy and fibrosis were established. On the 54th week, pancreatic glandular atrophy was observed (Fig. 2F and 2G). In addition, pancreatic acinar cells decreased in number and were replaced with interstitial tissues. In spite of the severe pancreatic degeneration, neoplastic changes were not observed. To evaluate pancreatic fibrosis, we performed azan staining. Although fibrous tissue around the TC was stained, the extent of the fibrosis was mild (Fig. $2 \mathrm{H}$ and 2I).

The number of TCs was $6.0 \pm 1.4$ at 12 weeks, $11.6 \pm$
1.7 at 16 weeks, $25.2 \pm 4.2$ at 24 weeks, $26.4 \pm 2.0$ at 32 weeks and $33.6 \pm 5.6$ at 54 weeks after starting the CDE diet; TCs were not observed in the control diet group (Fig. 3A). One-way ANOVA, indicated statistically significant increases in the number of TCs at 24,32 , and 54 weeks compared with the number at 4 weeks. In addition, we quantified the extent of acinar cell loss by morphometric analysis. In the control diet group, there was no significant decrease in acinar cell volume, although there was a slight decrease because of the increase in islet volume. In CDE-fed mice, the volume of acinar cells decreased gradually from $47 \pm 6 \%$ at 24 weeks to $44 \pm 16 \%$ at 32 weeks and $23 \pm 5 \%$ after 54 weeks of CDE diet (Fig. 3B).

\section{Activation of carcinogenesis-related molecules after long} term CDE diet

We investigated the molecular events caused by longterm CDE diet using western blotting. EGFR overexpression, EGFR phosphorylation and K-Ras gene muta- 

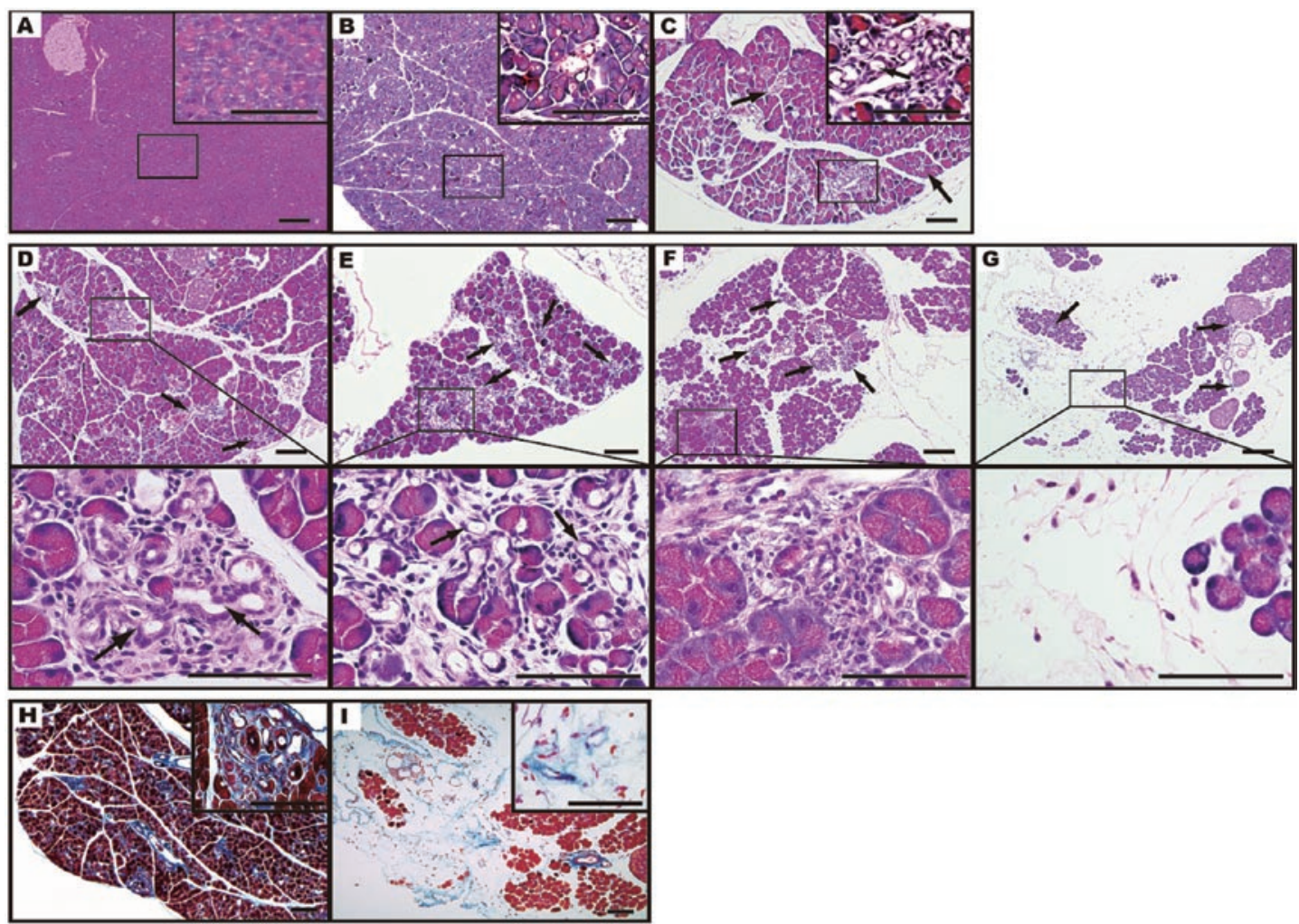

Fig. 2. Histological changes of the pancreas after long term $\mathrm{CDE}$ diet treatment. Mice were given regular laboratory chow (A) or CDE diet for 4 (B), 16 (C), 24 (D, E), or 54 (F, G) weeks. Pancreatic sections were analyzed by HE staining. Higher magnifications of the boxed areas at 24 and 54 weeks are shown in the lower column. Azan staining of pancreata from CDE diet mice is shown in $\mathrm{H}$ ( 24 weeks) and I ( 54 weeks). Arrows indicate tubular complexes. Scale bars: $100 \mu \mathrm{m}$.

(A)

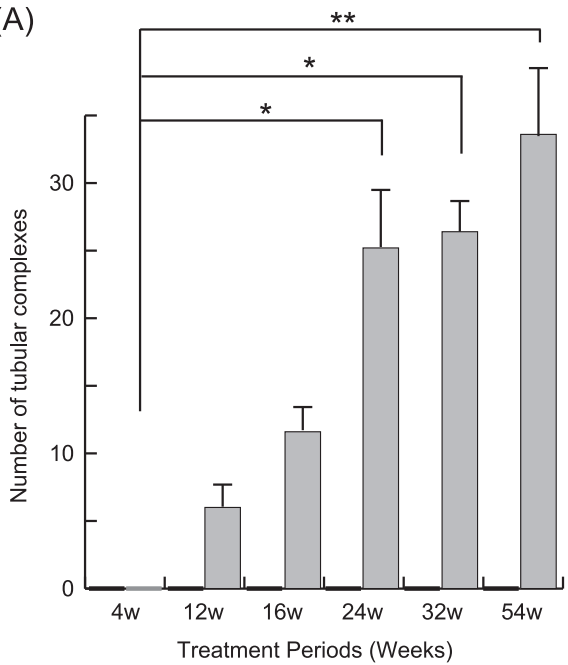

(B)

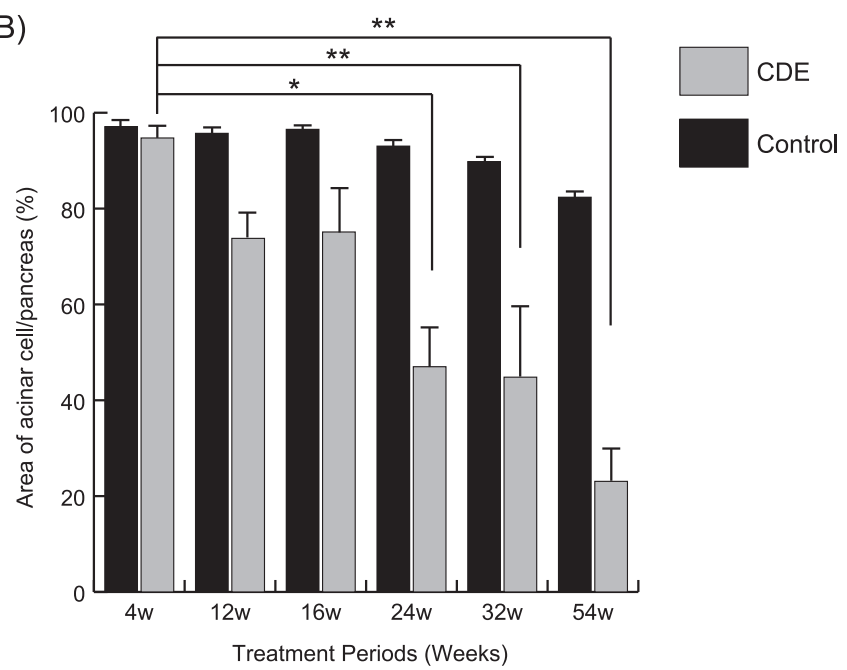

Fig. 3. Analysis of the number of tubular complexes and the volume of acinar cells. Number of tubular complexes (A) and volume of acinar cells (B) are shown for each treatment period. The black bar shows the control diet group, and the gray bar shows the CDE diet group. Data represent mean \pm SE. $\left(n=3,5\right.$ cuts per mouse, $\left.{ }^{* *} P<0.01,{ }^{*} P<0.05\right)$. 
tions (activation of K-Ras molecule) are reported to be the key events in the process of pancreatic carcinogenesis. In the control diet group, there were no significant changes during the examination period (Fig. 4A). Compared with the control diet group, CDE diet induced overexpression and phosphorylation of EGFR after 4 weeks (Fig. 4B). The level of EGFR expression reached its peak at 12 to 16 weeks and declined after 32 weeks. Activation of the K-Ras molecule was also detected after 12 weeks. Expression patterns of EGF, TGF $\alpha$, and Spink3, one of the potent ligands for EGFR [34], were similar to that of EGFR. The expression of EGF followed a similar course, but was weaker than that of the other ligands for EGFR. We also investigated the expression of Cox 2 as a parameter of inflammatory responses. Cox 2 was also overexpressed in a manner similar to EGFR in the mice fed the long-term CDE diet. There were no significant changes in amylase and trypsinogen levels related to the CDE diet (Fig. 4).

\section{Discussion}

In this study, we developed a model of chronic pancreatitis that reproduces three key responses of human chronic pancreatitis: development of acinar atrophy, fibrosis, and TCs. We also demonstrated the expression of EGFR and its potent ligands, phosphorylation of EGFR, and the activation of K-Ras in the step of chronic inflammation.

Compared with other experimental models, CDE dietinduced pancreatitis is considered to be an ideal model because of its similar natural history to that of the human disease as well as histological and biochemical similarities [2]. The mechanism of acute pancreatitis development in the mice fed the CDE diet remains unclear. It is likely that ethionine disrupts phospholipid metabolism of membranes that are involved in the processes of intracellular transport and secretion of pancreatic enzymes. A choline-deficient diet would potentiate the activity of ethionine in this respect, because a cholinedeficient diet also induces changes in membrane phospholipids of cellular organelles $[8,23,24]$. Thus, normal luminal exocytosis appeared to be blocked, and subsequently zymogen granules accumulated in the cytoplasm $[14,18]$. These changes may result in activation of zy- mogens eventually leading to pancreatitis. In any case, the CDE-diet model is simple and easy to prepare, and thus seems to be a promising model for studies of chronic pancreatitis.

As far as we know, this is the first report on the expression of carcinogenesis-related molecules such as EGFR, $\mathrm{K}-\mathrm{Ras}, \operatorname{Cox} 2$, and TGF $\alpha$, during chronic pancreatitis. Their expression started to increase at 4 weeks and reached their peaks at 12 and 16 weeks in the CDE diet model. Expression of these molecules decreased after 32 weeks. This may have been due to the decrease in the number of normally functioning pancreatic cells as demonstrated in Fig. 3B. EGFR and its ligand, TGF $\alpha$, as well as PDA, are frequently overexpressed in human pancreatic tissues obtained from patients with chronic pancreatitis [20,34]. The rate of $K$-Ras gene mutation increases along with disease progression in chronic pancreatitis, and the mutations are found in nearly $100 \%$ of PDA [3]. Furthermore, EGFR induction occurs in lowgrade pancreatic intra-epithelial neoplasias, indicating that autocrine EGF-family growth signaling is operative at the earliest stage of pancreatic neoplasia [3]. Nevertheless, PDA did not develop in our model, even after 54 weeks. On the other hand, TCs were formed with advancing age. TCs are considered to be precursor lesions of PDA [15, 35], and they have often been observed in human/rodent chronic pancreatitis and PDA [5, 6, 12, $15,36,37]$. There is one report in which the transition from chronic inflammation to metaplasia/dysplasia is described [9]. Colby et al. [9] reported that Cox 2 overexpression driven by the bovine keratin 5 promoter led to acinar-to-ductal metaplasia, increased proliferation of metaplastic ductal cells, and nuclear pleomorphisms accompanied the elevation of prostaglandin $\mathrm{E}_{2}\left(\mathrm{PGE}_{2}\right)$. Because $\mathrm{PGE}_{2}$ confers both survival and growth advantages, it may act as an inhibitor of apoptosis or a promoter of proliferation. We demonstrated the overexpression of Spink3 along with EGFR in western blot analysis. We previously demonstrated that the human serine protease inhibitor, Kazal type 1 (SPINK1: mouse homolog Spink3) can bind to EGFR and work as a growth factor through EGFR [34]. Thus, Spink3 may act as a growth factor, leading to cell proliferation and regeneration of tissue structure. In any case, chronic pancreatitis itself is not sufficient for the development 
(A)

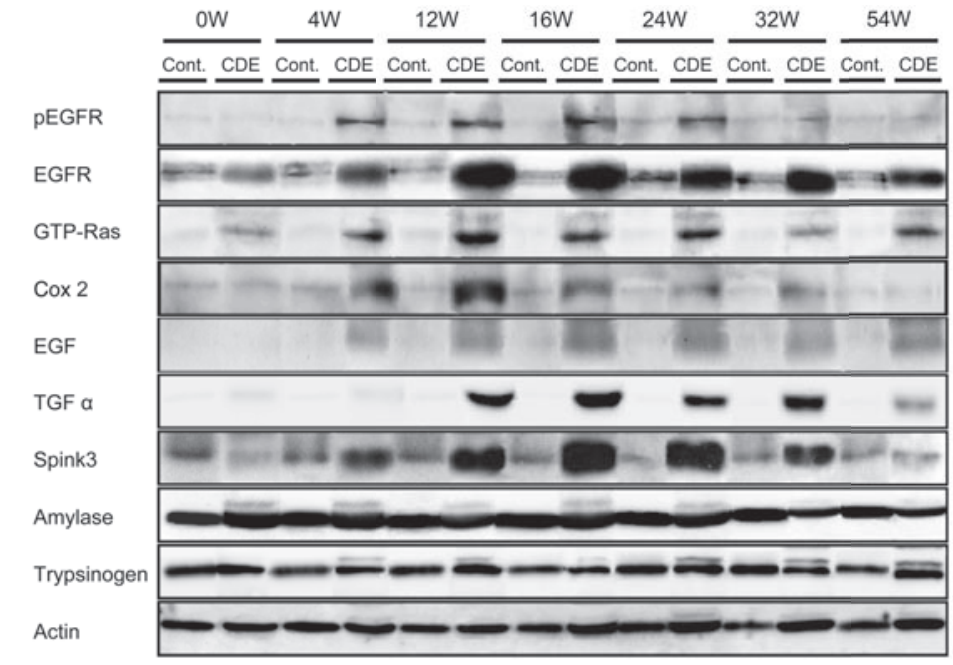

(B)
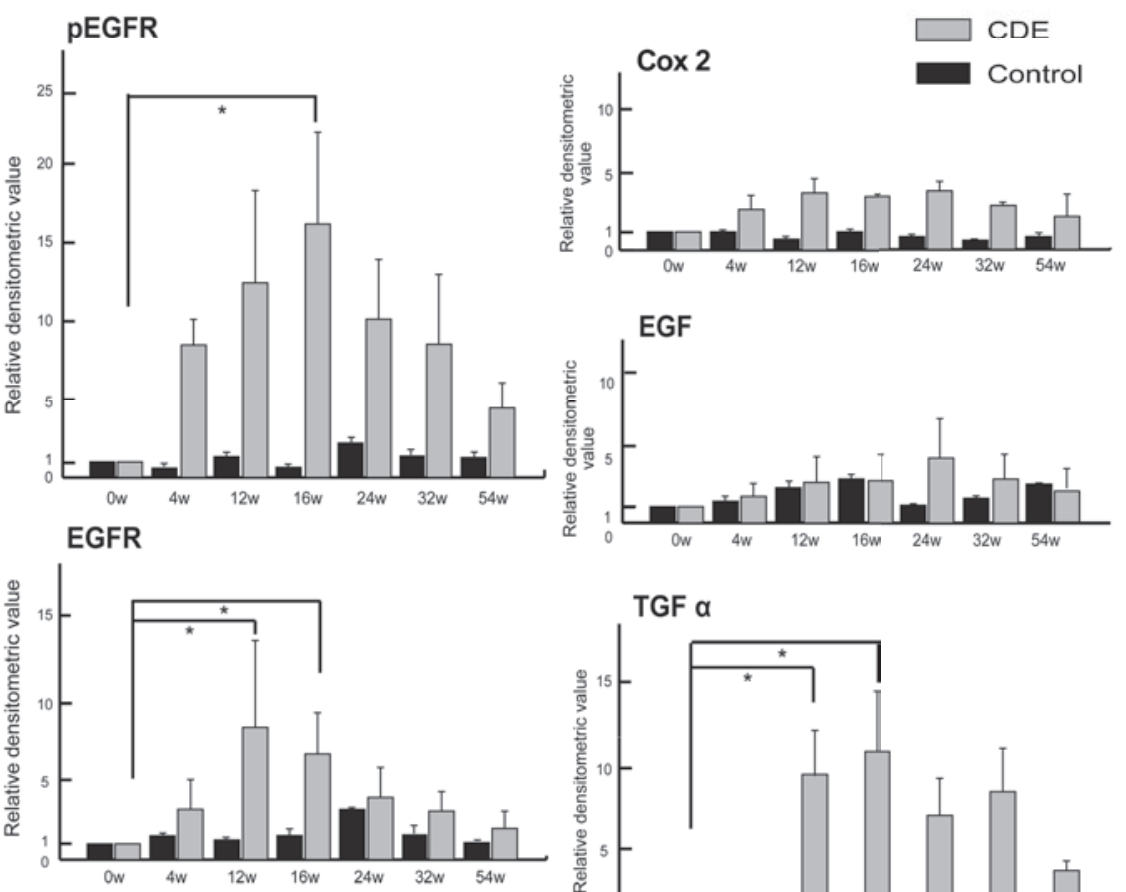

$$
\text { GTP-Ras }
$$
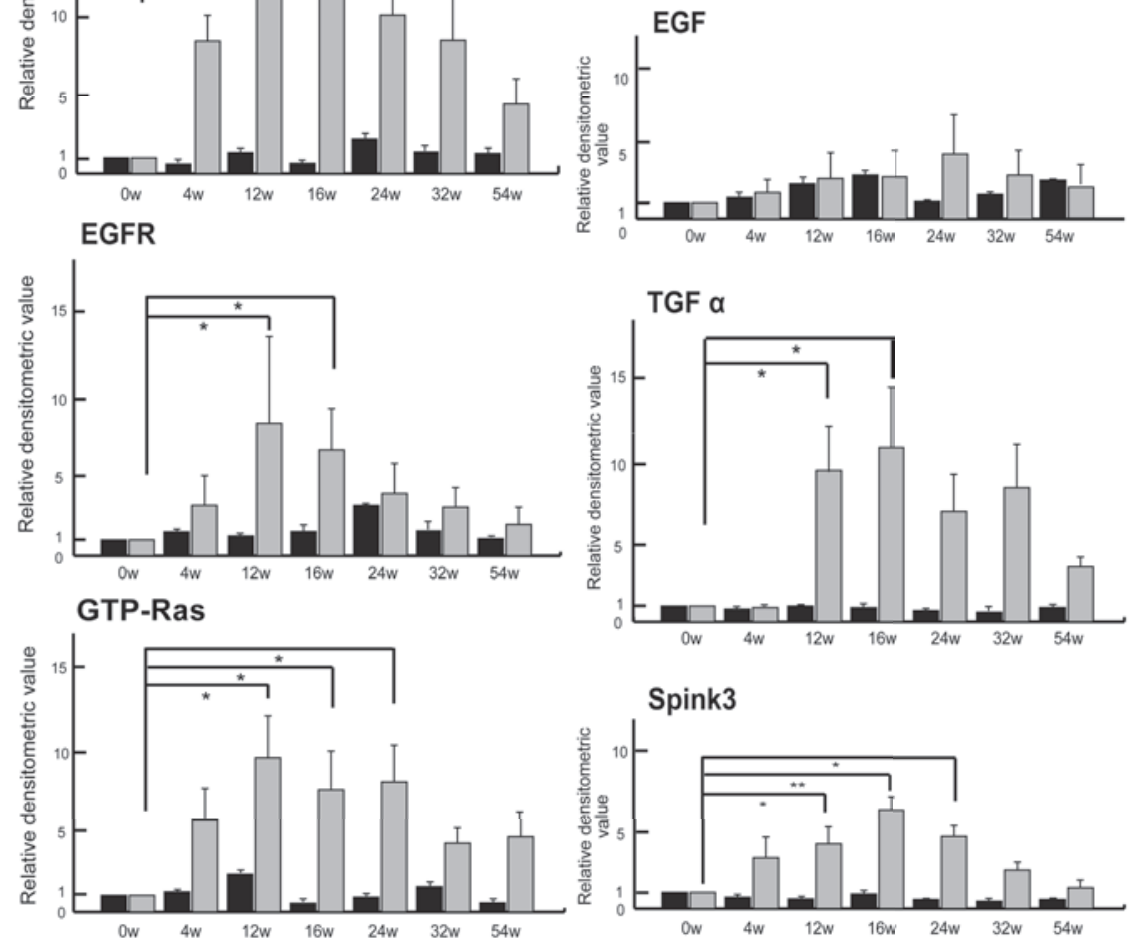

Fig. 4. Expression pattern of inflammation and/or carcinogenesis-related molecules. A. Western blot analysis was performed using the antibodies as described in Materials and Methods. B. Densitometric analysis for pEGFR, EGFR, GTP-Ras, Cox 2, TGF $\alpha$, and Spink3 before and after CDE diet by western blot. Data are expressed as the magnification of activity in the control before CDE diet after normalization by the actin signals. Only the data with significant differences are shown. Cont.: control diet group. CDE: CDE diet group. EGFR; epidermal growth factor receptor, pEGFR; phosphorylated EGFR, GTP-Ras; activated K-Ras, Cox 2; cycloox ygenase 2, EGF; epidermal growth factor, TGF $\alpha$; transforming growth factor $\alpha$, Spink 3 ; serine protease inhibitor, Kazal type $3\left(\mathrm{n}=3,{ }^{* *} P<0.01,{ }^{*} P<0.05\right)$. 
of PDA. Further combinations of CDE diet with the administration of other promotion insults or genetic alterations may result in PDA development.

\section{Acknowledgments}

We wish to thank Ms. Yumi Ohtake for her technical assistance. This work was supported by a Grant-in-Aid for Scientific Research Priority Areas (KAKENHI) (17012018) and a Grant-in Aid for Scientific Research (S) (21220010) from Japan Society for the Promotion of Science (JSPS).

\section{References}

1. Archer, H., Jura, N., Keller, J., Jacobson, M., and Bar-Sagi, D. 2006. A mouse model of hereditary pancreatitis generated by transgenic expression of $\mathrm{R} 122 \mathrm{H}$ trypsinogen. Gastroenterology 131: 1844-1855.

2. Banerjee, A.K., Galloway, S.W., and Kingsnorth, A.N. 1994. Experimental models of acute pancreatitis. Br. J. Surg. 81: 1096-1103.

3. Bardeesy, N. and DePinho, R.A. 2002. Pancreatic cancer biology and genetics. Nat. Rev. Cancer 2: 897-909.

4. Barton, C.M., Hall, P.A., Hughes, C.M., Gullick, W.J., and Lemoine, N.R. 1991. Transforming growth factor alpha and epidermal growth factor in human pancreatic cancer. $J$. Pathol. 163: 111-116.

5. Bockman, D.E., Boydston, W.R., and Anderson, M.C. 1982. Origin of tubular complexes in human chronic pancreatitis. Am. J. Surg. 144: 243-249.

6. Bockman, D.E., Guo, J., Buchler, P., Muller, M.W., Bergmann, F., and Friess, H. 2003. Origin and development of the precursor lesions in experimental pancreatic cancer in rats. Lab. Invest. 83: 853-859.

7. Callicutt, C.S., Sabek, O., Fukatsu, K., Lundberg, A.H., Gaber, L., Wilcox, H., Kotb, M., and Gaber, A.O. 2003. Diminished lung injury with vascular adhesion molecule-1 blockade in choline-deficient ethionine diet-induced pancreatitis. Surgery 133: 186-196.

8. Chen, S.H., Estes, L.W., and Lombardi, B. 1972. Lecithin depletion in hepatic microsomal membranes of rats fed on a choline-deficient diet. Exp. Mol. Pathol. 17: 176-186.

9. Colby, J.K., Klein, R.D., McArthur, M.J., Conti, C.J., Kiguchi, K., Kawamoto, T., Riggs, P.K., Pavone, A.I., Sawicki, J., and Fischer, S.M. 2008. Progressive metaplastic and dysplastic changes in mouse pancreas induced by cyclooxygenase-2 overexpression. Neoplasia 10: 782796.

10. Day, J.D., Digiuseppe, J.A., Yeo, C., Lai-Goldman, M., Anderson, S.M., Goodman, S.N., Kern, S.E., and Hruban, R.H. 1996. Immunohistochemical evaluation of HER-2/neu expression in pancreatic adenocarcinoma and pancreatic intraepithelial neoplasms. Hum. Pathol. 27: 119-124.
11. Durie, P.R., Kent, G., Phillips, M.J., and Ackerley, C.A. 2004. Characteristic multiorgan pathology of cystic fibrosis in a long-living cystic fibrosis transmembrane regulator knockout murine model. Am. J. Pathol. 164: 1481-1493.

12. Esposito, I., Seiler, C., Bergmann, F., Kleeff, J., Friess, H., and Schirmacher, P. 2007. Hypothetical progression model of pancreatic cancer with origin in the centroacinar-acinar compartment. Pancreas 35: 212-217.

13. Friess, H., Berberat, P., Schilling, M., Kunz, J., Korc, M., and Buchler, M.W. 1996. Pancreatic cancer: the potential clinical relevance of alterations in growth factors and their receptors. J. Mol. Med. 74: 35-42.

14. Gilliland, L. and Steer, M.L. 1980. Effects of ethionine on digestive enzyme synthesis and discharge by mouse pancreas. Am. J. Physiol. 239: G418-426.

15. Jimenez, R.E., Z'Graggen, K., Hartwig, W., Graeme-Cook, F., Warshaw, A.L., and Fernandez-del Castillo, C. 1999 Immunohistochemical characterization of pancreatic tumors induced by dimethylbenzanthracene in rats. Am. J. Pathol. 154: 1223-1229.

16. Kihara, Y., Yoshikawa, H., Honda, H., Fukumitsu, K., Yamaguchi, T., and Otsuki, M. 2005. Natural disruption of group 2 phospholipase A2 gene protects against cholinedeficient ethionine-supplemented diet-induced acute pancreatitis and lung injury. Pancreas 31: 48-53.

17. Kloppel, G. and Maillet, B. 1993. Pathology of acute and chronic pancreatitis. Pancreas 8: 659-670.

18. Koike, H., Steer, M.L., and Meldolesi, J. 1982. Pancreatic effects of ethionine: blockade of exocytosis and appearance of crinophagy and autophagy precede cellular necrosis. Am . J. Physiol. 242: G297-307.

19. Korc, M., Chandrasekar, B., Yamanaka, Y., Friess, H., Buchier, M., and Beger, H.G. 1992. Overexpression of the epidermal growth factor receptor in human pancreatic cancer is associated with concomitant increases in the levels of epidermal growth factor and transforming growth factor alpha. J. Clin. Invest. 90: 1352-1360.

20. Korc, M., Friess, H., Yamanaka, Y., Kobrin, M.S., Buchler, M., and Beger, H.G. 1994. Chronic pancreatitis is associated with increased concentrations of epidermal growth factor receptor, transforming growth factor alpha, and phospholipase C gamma. Gut 35: 1468-1473.

21. Lampel, M. and Kern, H.F. 1977. Acute interstitial pancreatitis in the rat induced by excessive doses of a pancreatic secretagogue. Virchows Arch. A Pathol. Anat. Histol. 373: 97-117.

22. Lechene de la Porte, P., Iovanna, J., Odaira, C., Choux, R., Sarles, H., and Berger, Z. 1991. Involvement of tubular complexes in pancreatic regeneration after acute necrohemorrhagic pancreatitis. Pancreas 6: 298-306.

23. Leelavathi, D.E., Katyal, S.L., and Lombardi, B. 1974. Lecithin depletion in liver mitochondria of rats fed a cholinedeficient diet. Effect on beta-hydroxybutyrate dehydrogenase. Life Sci. 14: 1203-1210.

24. Lombardi, B. 1971. Effects of choline deficiency on rat hepatocytes. Fed. Proc. 30: 139-142.

25. Lowenfels, A.B., Maisonneuve, P., Cavallini, G., Ammann, R.W., Lankisch, P.G., Andersen, J.R., Dimagno, E.P., 
Andren-Sandberg, A., and Domellof, L. 1993. Pancreatitis and the risk of pancreatic cancer. International Pancreatitis Study Group. N. Engl. J. Med. 328: 1433-1437.

26. Lowenfels, A.B., Maisonneuve, P., DiMagno, E.P., Elitsur, Y., Gates, L.K. Jr., Perrault, J., and Whitcomb, D.C. 1997. Hereditary pancreatitis and the risk of pancreatic cancer. International Hereditary Pancreatitis Study Group. J. Natl. Cancer Inst. 89: 442-446.

27. Malka, D., Hammel, P., Maire, F., Rufat, P., Madeira, I., Pessione, F., Levy, P., and Ruszniewski, P. 2002. Risk of pancreatic adenocarcinoma in chronic pancreatitis. Gut 51: 849-852.

28. Marrache, F., Tu, S.P., Bhagat, G., Pendyala, S., Osterreicher, C.H., Gordon, S., Ramanathan, V., Penz-Osterreicher, M., Betz, K.S., Song, Z., and Wang, T.C. 2008. Overexpression of interleukin-1beta in the murine pancreas results in chronic pancreatitis. Gastroenterology 135: 1277-1287.

29. Neuschwander-Tetri, B.A., Burton, F.R., Presti, M.E., Britton, R.S., Janney, C.G., Garvin, P.R., Brunt, E.M., Galvin, N.J., and Poulos, J.E. 2000. Repetitive self-limited acute pancreatitis induces pancreatic fibrogenesis in the mouse. Dig. Dis. Sci. 45: 665-674.

30. Niederau, C., Luthen, R., Niederau, M.C., Grendell, J.H., and Ferrell, L.D. 1992. Acute experimental hemorrhagicnecrotizing pancreatitis induced by feeding a cholinedeficient, ethionine-supplemented diet. Methodology and standards. Eur. Surg. Res. 24: 40-54.

31. Ohmuraya, M., Hirota, M., Araki, M., Mizushima, N., Matsui, M., Mizumoto, T., Haruna, K., Kume, S., Takeya, M., Ogawa, M., Araki, K., and Yamamura, K. 2005. Autophagic cell death of pancreatic acinar cells in serine protease inhibitor Kazal type 3-deficient mice. Gastroenterology 129: 696-705.

32. Oikawa, T., Hitomi, J., Kono, A., Kaneko, E., and Yamaguchi,
K. 1995. Frequent expression of genes for receptor tyrosine kinases and their ligands in human pancreatic cancer cells. Int. J. Pancreatol. 18: 15-23.

33. Overholser, J.P., Prewett, M.C., Hooper, A.T., Waksal, H.W., and Hicklin, D.J. 2000. Epidermal growth factor receptor blockade by antibody IMC-C225 inhibits growth of a human pancreatic carcinoma xenograft in nude mice. Cancer 89 : 74-82.

34. Ozaki, N., Ohmuraya, M., Hirota, M., Ida, S., Wang, J., Takamori, H., Higashiyama, S., Baba, H., and Yamamura, K. 2009. Serine protease inhibitor, Kazal type 1, promotes proliferation of pancreatic cancer cells through the epidermal growth factor receptor. Mol. Cancer Res. (in press).

35. Parsa, I., Longnecker, D.S., Scarpelli, D.G., Pour, P., Reddy, J.K., and Lefkowitz, M. 1985. Ductal metaplasia of human exocrine pancreas and its association with carcinoma. Cancer Res. 45: 1285-1290.

36. Strobel, O., Dor, Y., Alsina, J., Stirman, A., Lauwers, G., Trainor, A., Castillo, C.F., Warshaw, A.L., and Thayer, S.P. 2007. In vivo lineage tracing defines the role of acinar-toductal transdifferentiation in inflammatory ductal metaplasia. Gastroenterology 133: 1999-2009.

37. Wagner, M., Luhrs, H., Kloppel, G., Adler, G., and Schmid, R.M. 1998. Malignant transformation of duct-like cells originating from acini in transforming growth factor transgenic mice. Gastroenterology 115: 1254-1262.

38. Watanabe, M., Nobuta, A., Tanaka, J., and Asaka, M. 1996. An effect of K-ras gene mutation on epidermal growth factor receptor signal transduction in PANC-1 pancreatic carcinoma cells. Int. J. Cancer 67: 264-268.

39. Willemer, S. and Adler, G. 1989. Histochemical and ultrastructural characteristics of tubular complexes in human acute pancreatitis. Dig. Dis. Sci. 34: 46-55. 